\title{
Welcome to the Special Section on Digital Museum Technology
}

\author{
Guest Editors: Kiyoharu Aizawa and Michitaka Hirose
}

Recent progress in digital technology made media technology applicable to museums which have been the places, for a long period of time, where people meet physical social archives. Museums show not only physical objects, but also events in the past. They have relied on traditional way of exhibition which does not include information technology. Digital technology could improve existing museums in two ways. One is advancing visitors' experiences by introducing information technology in the exhibitions such as mixed reality. The other is improvement of management of museums by monitoring visitors so that new aspects of visitors' behaviors could be found. Thus, there are many potential research topics in digital museum technology.

In this special section, we have received thirteen papers and finally accepted seven papers. All the papers in this issue focus on new technology of exhibitions.

We are very grateful to all the authors who have submitted their excellent works to this issue as well as to all associate editors and the anonymous reviewers. We hope this special section will promote research in the field of digital museum technology.

MTA Editorial Committee members list (Vol.2, No.3, 2014)

\section{Guest Editors}

Kiyoharu Aizawa

\section{Associate Editors}

Tomohiro Tanikawa

Takeshi Naemura

Takeshi Oishi

Takuji Narumi
The University of Tokyo

The University of Tokyo

The University of Tokyo

The University of Tokyo

The University of Tokyo
Michitaka Hirose

The University of Tokyo

Hiromi Tanaka

Naomi Inoue

Ritsumeikan University

Yuichi Iwadate

NHK 